\title{
Virtual Campus Hub: Toward a concept for international collaboration in education
}

\author{
Frank Vercoulen ${ }^{1}$ \\ ${ }^{1}$ Eindhoven University of Technology, PO Box 513, 5600 MB Eindhoven, The Netherlands, \\ f.j.h.m.vercoulen@tue.nl.
}

\section{Keywords}

International collaboration, online collaboration, federated authentication, group management, portals, Surfconext, Géant, eduGAIN.

\section{ABSTRACT}

Virtual Campus Hub is a European FP7 project, which aims to remove some important IT barriers for international collaboration in education (e.g. joint programs). Examples are seamless access to partners' learning materials and document sharing environments. To achieve this, Virtual Campus Hub makes use of the European Géant infrastructure, based on national NREN federations interconnected through the inter-federation eduGAIN. To demonstrate the feasibility of the Virtual Campus Hub, a demo portal has been set up, which provides single-sign on access to partners' applications and which makes use of Surfnet's group management module Surfteams to manage access for international groups. Partners in Virtual Campus Hub are Technical University of Denmark (DTU), Royal Institute of Technology Stockholm (KTH), Politecnico di Torino (PoliTo) and Eindhoven University of Technology (TU/e), as well as the corresponding federations (Surfconext NL, WAYF DK, Swamid SE and IDEM IT).

\section{OVERVIEW}

Virtual Campus Hub (VCH, http://www.virtualcampushub.eu) is a European 7th Framework project with Technical University of Denmark (DTU), Royal Institute of Technology, Sweden (KTH), Politecnico di Torino, Italy (PoliTo) and Eindhoven University of Technology, The Netherlands (TU/e) as partners. In this project, we try to find out how to remove some important IT barriers for international collaboration in education, research and innovation.

Every institution has a well-functioning and easily accessible digital learning and working environment for students and teachers. However, once an institution's borders are crossed, e.g. in a joint program of European universities (but collaboration with industry and between universities and secondary schools are good examples as well), this easy access turns into a complex jumble of information channels and applications, for which you also need several accounts to access them. As a result, information is often unreliable and students and teachers lose way too much time with getting access to applications and information and with organizing their communication with teachers and peers. Standardizing on a single platform for all partners is no solution, as institutions are involved in many other collaborations as well (which may have different preferences) and standardization is very difficult to achieve anyway.

In Virtual Campus Hub, we investigate, both by setting up a pilot environment and by conceptually working out use cases for concrete joint educational programs, how some of the most important barriers for international collaboration could be removed and which of these barriers should have priority. The pilot environment, which is expected to be ready in May 2013, and which will serve as a technical proof of concept, will consist of the following:

- Login with your own account to educational applications at a partner university

- Enable easier access to interactive learning materials at a partner university

- Enable easier access to collaboration environments at a partner university

- More efficient and more flexible organization of online meetings, distance lectures and online coaching. 
- Easy access to all relevant applications at partner universities through a simple (single signon) demo portal

The conceptual use case for a concrete joint program, which is expected to be ready in October 2013, will also deal with other aspects of a digital learning and working environment, such as retrieval of course and scheduling information across universities, retrieval of learning results and retrieval of news and other important information on one's study program.

\section{TECHNICAL CONCEPT}

The technical concept for Virtual Campus Hub relies heavily on the facilities offered by the European Géant infrastructure and its organizational structure based on national federations (the so-called NREN federations) interconnected through the inter-federation eduGAIN (http://www.edugain.eu). In this project, the NREN federations from the Netherlands (Surfnet), Denmark (Wayf), Sweden (Swamid) and Italy (IDEM) are involved.

Virtual Campus Hub makes use of two important infrastructure functionalities:

1. (federated authentication) Log in with the account of your own institution to all relevant applications (also those of other partners). Applications need to be enabled to make use of federated authentication and must be connected to their national federation.

2. (group management) Create and manage cross-institutional groups. All applications can use this group information for authorization. This enables easy access and considerably lowers the administrative load to provide access to every different application separately. Applications need to be enabled to make use of external group management information and must be connected to a general group management application, in this project SURFteams provided by the Dutch NREN Surfnet and part of its federation Surfconext (http://www.surfconext.nl). The reason to choose this application is that there is no standardized application available yet across NRENs that would be able to provide this functionality in a generalized way. However, Surfconext is also available as open source, OpenConext (http://www.openconext.org) and at the NREN and Géant level work is being done to harmonize protocols with respect to group management, so a structural solution is expected to become available in some time.

Access to all functions is realized through a simple (single sign-on) demoportal, which also offers the possibility to manage international groups through SURFteams, in the future to be replaced by a general (European?) solution for group management.

\section{RESULTS}

The results of this project will be:

- Insight in the technical, organizational and pedagogical aspects of realizing international collaboration in education (e.g. for joint educational programs).

- Experience with using the available facilities from the Géant infrastructure for collaboration in education and an overview of additional infrastructure functionalities needed for international collaboration in education.

\section{AUTHORS' BIOGRAPHIES}

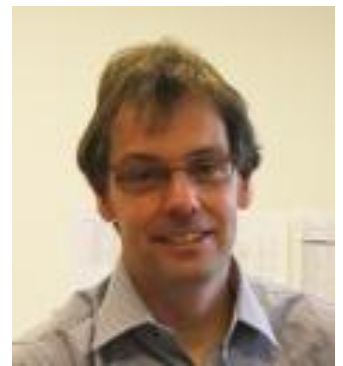

Frank Vercoulen is the manager of the education portal environment at Eindhoven University of Technology (TU/e) and has been involved in a number of national and international projects on supporting cross-institutional collaboration in education. He has a master degree in physics from the University of Utrecht. 